\title{
application du calcul à la rupture à la stabilité du front de taille d'un tunnel
}

\author{
analysis of tunnel front stability, \\ using the yield calculation approach
}

\author{
E. LECA \\ C.E.R.M.E.S. * \\ M. PANET \\ Professeur adjoint à l'E.N.P.C., SIMECSOL *
}

\section{Résumé}

La réalisation d'un tunnel dans de bonnes conditions de sécurité nécessite un examen préalable de la stabilité du front de taille pendant l'excavation. Lorsque l'ouvrage est creusé à faible profondeur et à l'aide d'une machine foreuse permettant un soutènement continu du front de taille, il faut tenir compte dans l'analyse, de trois effets de chargement: influence du poids des terrains, surcharges de surface, pression de soutènement au front.

L'approche par le calcul à la rupture est choisie ici pour estimer les conditions de stabilité du front de taille. Deux types de matériau sont considérés: dans le cas d'un matériau purement cohérent (critère de TRESCA) la combinaison de l'approche par l'intérieur et de l'approche par l'extérieur permet d'aboutir à un encadrement des conditions de stabilité dans un diagramme de chargement à deux paramètres. Dans le cas plus complexe d'un critère de MOHR-COULOMB, il est nécessaire de prendre effectivement en compte trois paramètres de chargement et seule l'approche par l'intérieur est considérée dans la présente étude.

\section{Abstract}

Front stability must be examined prior to tunnel construction in order to ensure good safety conditions while boring it. When the tunnel is at shallow depth and is driven using a boring machine with full face support, three loading effects need to be considered: influence of the soil weight, soil surface loads, retaining pressure at the front.

The yield calculation approach is used in this paper to estimate stability conditions for the tunnel front. Two material types are considered: in the case of a purely cohesive material (TRESCA yield criterion) combining lower bound and upper bound analyses leads to bracketting stability conditions for the front with respect to two loading parameters only. In the more difficult case of a MOHR-COULOMB criterion however, it is necessary to account for three loading parameters; for this latter material type the lower bound approach only is considered in the present study.

- École nationale des Ponts et Chaussées, La Courtine, 93167 Noisy le Grand Cedex.

$\because 8$, avenue Newton, 92350 Le Plessis-Robinson. 


\section{INTRODUCTION}

De nombreux travaux de recherche ont été consacrés à la stabilité du front de taille d'un tunnel au cours des vingts dernières années. Il s'agit d'études à caractère aussi bien expérimental que théorique, mais qui le plus souvent se limitent au cas des terrains argileux.

La présente étude a pour objet d'examiner l'approche par le calcul à la rupture, pour un tunnel à section circulaire revêtu sur toute sa longueur par un ouvrage parfaitement rigide. Le front de taille est supposé être maintenu en équilibre par une pression interne uniforme. Une telle pression correspond dans la pratique au système de soutènement mis en cuvre sur les machines modernes d'excavation : bouclier à air comprimé, à boue bentonitique, à pression de terre.

Après avoir précisé le problème d'un point de vue analytique, les cas d'un terrain purement cohérent (critère de TRESCA) et d'un matériau frottant-cohérent (critère de MOHR-COULOMB) sont étudiés successivement.

Le paragraphe 3 constitue un approfondissement des résultats obtenus par E.H. DAVIS, M.J. GUNN, R.J. MAIR, H.N. SENEVIRATNE (1980) pour une argile. Le même type d'analyse est généralisé au cas d'un critère de MOHR-COULOMB au paragraphe 4 , dans le but de développer une approche par l'intérieur en terrain frottant-cohérent.

\section{POSITION DU PROBLÈME}

Du fait de la présence du front de taille, la stabilité de la section en cours d'excavation d'un tunnel constitue un problème tridimensionnel vrai, appelé problème ( $\mathrm{Pb} \mathrm{I})$ dans la suite. Le modèle analytique adopté est précisé dans les paragraphes qui suivent. Une telle étude s'avère généralement difficile et on est souvent conduit, dans une première approche à considérer deux problèmes plans : le long de l'axe du tunnel d'une part $(\mathrm{Pb}$ II), en section courante d'autre part ( $\mathrm{Pb}$ III). Ces deux cas de figure sont décrits au paragraphe 2.4 .

\subsection{Données et hypothèses pour le problème tridimensionnel}

On considère un tunnel cylindrique horizontal (figure 2.1.1.), à section circulaire, de diamètre D. La couverture est égale à $C$ et par conséquent la profondeur est :

$H=C+\frac{D}{2}$

Le tunnel est revêtu par un ouvrage supposé infiniment rigide et seule une région de longueur $\mathrm{P}$ derrière le front de taille n'est pas revêtue.

Une pression interne de soutènement $\sigma_{\mathrm{T}}$ est appliquée dans cette région et la surface du sol est chargée par une pression de surface $\sigma_{s}$ qui peut représenter la charge transmise par une fondation souple ou encore le poids d'une couche de sol située au-dessus de la cote $y=H$. On s'intéressera essentiellement ici au cas où $\mathrm{P} / \mathrm{D}=0$ et où la pression $\sigma_{\mathrm{T}}$ s'applique uniquement au front de taille. La pression $\sigma_{T}$ est supposée uniforme sur toute la hauteur du front de taille.

La résistance du massif est caractérisée par la cohésion non drainée $C_{u}$ dans le cas d'un sol purement cohérent (critère de TRESCA), par la cohésion c' et l'angle de frottement $\varphi$ ' dans le cas d'un sol frottant-cohérent (critère de MOHR-COULOMB). Il est souvent commode d'introduire alors la résistance en compression simple :

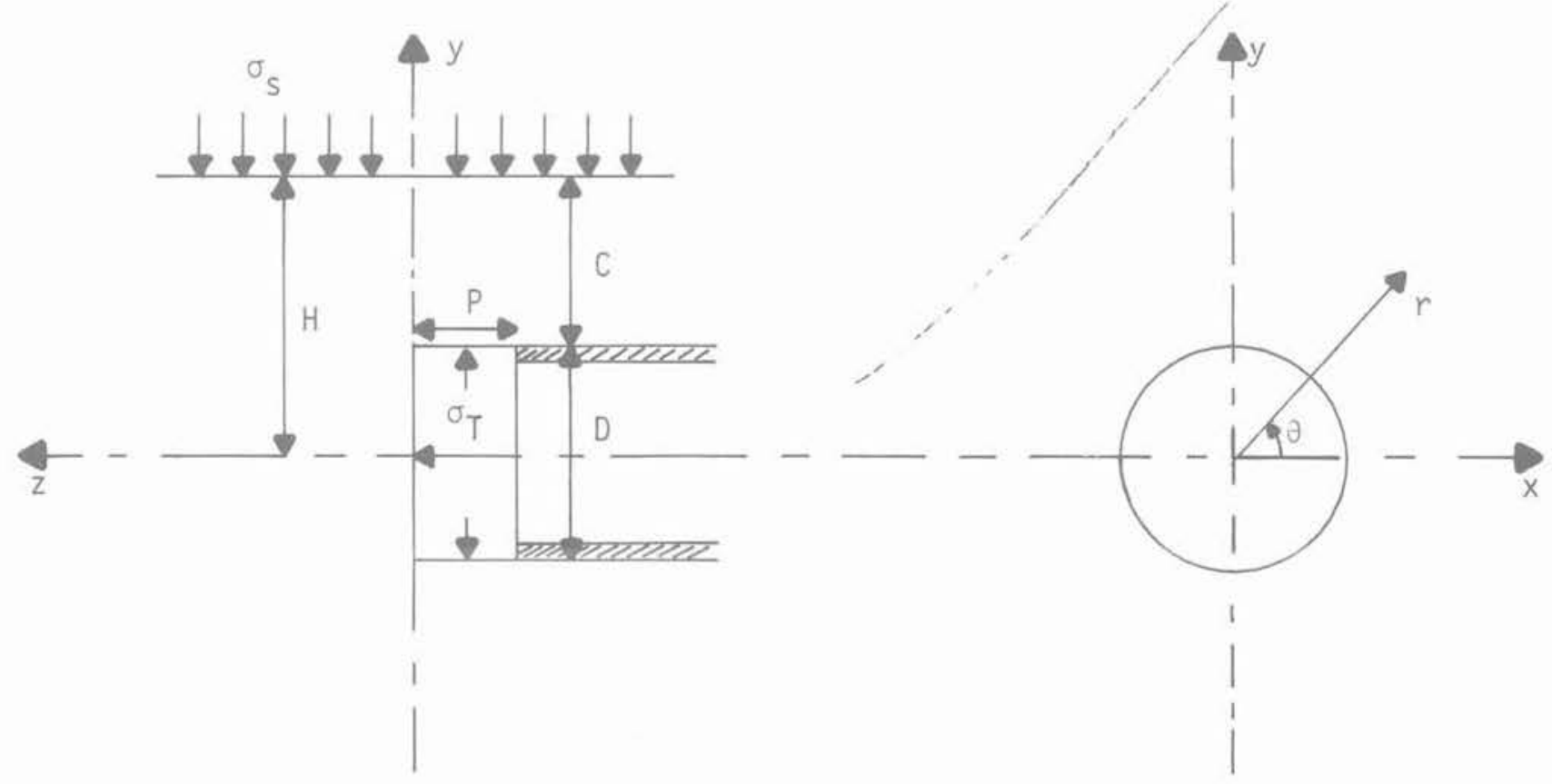

Fig. 2.1.1. - Géométrie du problème (Pb I).

Fig. 2.1.1. - Geometry for case (PD I). 


$$
\sigma_{c}=\frac{2 c^{\prime} \cos \varphi^{\prime}}{1-\sin \varphi^{\prime}}
$$

\subsection{Analyse dimensionnelle $(\mathrm{Pb} \mathrm{I})$}

Le problème présenté au paragraphe (2.1.) fait intervenir les grandeurs suivantes :

- longueur : D, C (ou H), P,

- pressions : $\sigma_{s}, \sigma_{\mathrm{T}}, \gamma_{\mathrm{D}}, \sigma_{\mathrm{c}}\left(\mathrm{ou} \mathrm{C}_{\mathrm{u}}\right)$,

- angle : $\varphi^{\prime}$.

Toute caractérisation de la stabilité du front de taille devra donc faire apparaître à priori les grandeurs adimensionnelles :

$-\frac{\mathrm{C}}{\mathrm{D}}, \frac{\mathrm{P}}{\mathrm{D}}, \frac{\gamma_{\mathrm{D}}}{\mathrm{Cu}}, \frac{\sigma_{\mathrm{s}}}{\mathrm{Cu}}, \frac{\sigma_{\mathrm{T}}}{\mathrm{Cu}}$ dans le cas d'un sol purement cohérent.

$-\frac{\mathrm{C}}{\mathrm{D}}, \frac{\mathrm{P}}{\mathrm{D}}, \frac{\gamma_{\mathrm{D}}}{\sigma_{\mathrm{c}}}, \frac{\sigma_{\mathrm{s}}}{\sigma_{\mathrm{c}}}, \frac{\sigma_{\mathrm{T}}}{\sigma_{c}}, \varphi^{\prime}$ dans le cas d'un sol frottant-cohérent.

\subsection{Les équations $(\mathrm{Pb} \mathrm{I})$}

Dans la suite on utilisera les notations et conventions suivantes :

_ $\underline{\sigma}$ désigne le tenseur des contraintes dans le massif; - Les contraintes sont positives en compression (conventions de la mécanique des sols);

- Les contraintes principales sont désignées par $\sigma_{1}$, $\sigma_{2}, \sigma_{3}$ avec $\sigma_{1} \geqslant \sigma_{2} \geqslant \sigma_{3}$;

- La contrainte normale $\sigma$ et la contrainte tangentielle $\tau$ sur un plan défini par le vecteur normal unitaire $n$ sont données par les relations,

$\sigma=\underline{n} \cdot \underline{\underline{g}} \cdot \underline{n}, /|\tau|=\sqrt{|\underline{g} \cdot \underline{n}|^{2}-(\underline{n} \cdot \underline{g} \cdot \underline{n})^{2}} ;$

- $\gamma$ désigne le vecteur poids volumique;

- Les coordonnées cartésiennes sont définies par $\mathrm{x}$,

$y, z$ (figure 2.1.1.), $\vec{x}, \vec{y}, \vec{z}$ désignant les vecteurs unitaires parallèles aux axes $\mathrm{Ox}, \mathrm{Oy}, \mathrm{Oz}$ respectivement;

- Les coordonnées polaires sont définies par le rayon $r$ et l'angle $\theta ; \vec{u}$. (resp. $\overrightarrow{\mathrm{u}_{\theta}}$ ) désigne le vecteur radial (resp. orthoradial) et $\mathrm{u}_{\mathrm{r}}$ (resp. $\mathrm{u}_{0}$ ) le déplacement suivant la direction radiale (resp. orthoradiale).

La résolution du problème de mécanique $(\mathrm{Pb}$ I) conduit à écrire :

- les équations d'équilibre :

$$
\operatorname{div} . \underline{\underline{g}}-\underline{y}=\underline{0}
$$

- les conditions aux limites :

$$
\underline{\underline{g}} \overrightarrow{\mathrm{y}}=-\sigma_{s} \overrightarrow{\mathrm{y}} \text { pour } \mathrm{y}=\mathrm{H}
$$

(surcharge $\sigma_{\mathrm{s}}$ en surface)

$$
\begin{gathered}
\stackrel{\sigma}{\underline{n}} n=\sigma_{\tau} \underline{n} \text { pour } \mathrm{r}=\frac{\mathrm{D}}{2} \text { et }-\mathrm{P} \leqslant \mathrm{z} \leqslant 0 \\
\stackrel{\sigma}{\underline{.}} \overrightarrow{\mathrm{z}}=\sigma_{\mathrm{T}} \overrightarrow{\mathrm{z}} \text { pour } \mathrm{r} \leqslant \frac{\mathrm{D}}{2} \text { et } \mathrm{z}=0
\end{gathered}
$$

(pression interne $\sigma_{T}$ au niveau du front de taille)

$$
u_{r}=0 \text { pour } r=\frac{D}{2} \text { et } z \leqslant-P
$$

La loi de comportement du terrain n'est à priori pas connue, mais on peut préciser le critère de résistance : - cas d'un matériau de TRESCA :

$$
\sigma_{1}-\sigma_{3} \leqslant 2 C_{u} \quad \text { (ou }|\tau| \leqslant 2 C_{u} \text { ) }
$$

- cas d'un matériau de MOHR-COULOMB

$$
\begin{gathered}
\sigma_{1}-\sigma_{3} \leqslant 2 c^{\prime} \cos \varphi^{\prime}+\left(\sigma_{1}+\sigma_{3}\right) \sin \varphi^{\prime} \\
\text { (ou } \left.|\tau| \leqslant c^{\prime}+\sigma^{\prime} \operatorname{tg} \varphi^{\prime}\right)
\end{gathered}
$$

On dispose ainsi de suffisamment d'information pour appliquer la théorie du calcul à la rupture (J. SALENÇON, 1979). Le système mécanique considéré ici est soumis à un nombre fini de paramètres de chargement et on cherchera à approcher au mieux le domaine (P) des chargements « potentiellement supportables ». La définition d'un champ de contrainte statiquement admissible et plastiquement admissible pour un chargement donné permettra d'affirmer que ce chargement est à l'intérieur du domaine (P). La mise en évidence d'un mécanisme de rupture permettra d'isoler un ensemble de points extérieurs à $(\mathrm{P})$. Contrairement à lanalyse limite, on parlera ici de champs « potentiellement supportables " plutôt que supportables, ce qui signifie qu'on ne dispose d'aucune certitude de stabilité pour un point intérieur à $(\mathrm{P})$. La stabilité ne peut être affirmée que sous certaines hypothèses sur la nature du matériau, qui ne seront pas considérées ici.

\section{4. Études bidimensionnelles}

Le problème $(\mathrm{Pb}$ II) est représenté sur la figure (2.4.1). On considère non plus une cavité cylindrique de diamètre $D$, mais une cavité de hauteur $D$, suffisamment

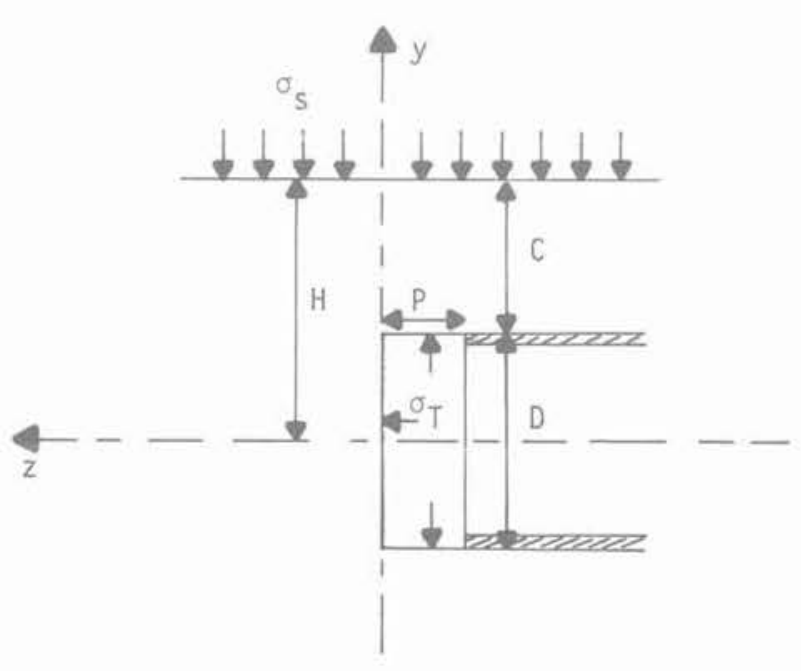

Fig. 2.4.1. - Géométrie du problème (Pb II). Fig. 2.4.1. - Geometry for case (Pb II). 
longue dans la direction $\mathrm{x}$ pour pouvoir se placer dans lhypothèse de déformations planes (pas de déformation suivant $\mathrm{x}$ ).

Le problème ( $\mathrm{Pb}$ III) (figure 2.4.2) est également plan, les déformations étant cette fois nulles dans la direction $\mathrm{OZ}$. On considère donc une cavité circulaire de diamètre $\mathrm{D}$ soumise à une pression interne uniforme $\sigma_{\mathrm{T}}$.

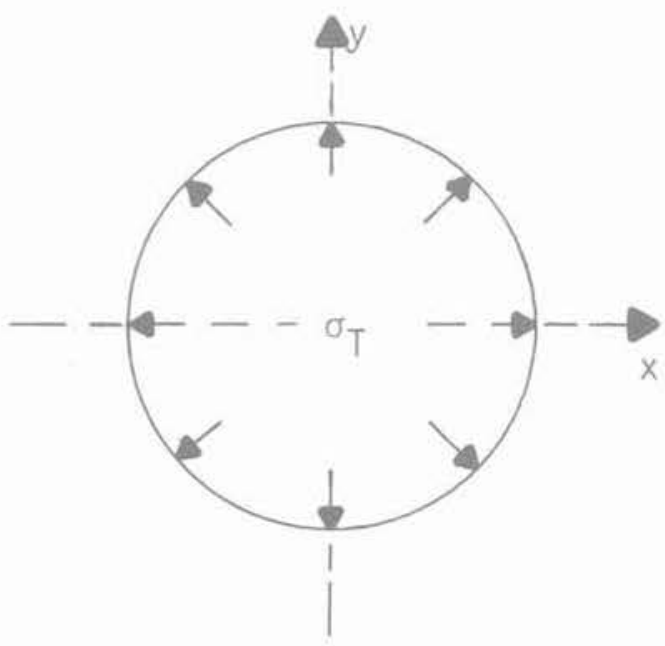

Fig. 2.4.2. - Géométrie du problème (Pb III). Fig. 2.4.2. - Geometry for case (Pb III).

Pour une section donnée, $\sigma_{\mathrm{T}}$ représente la pression exercée par l'ouvrage de soutènement, qui par conséquent est supposée uniforme. Bien que le champ de contrainte soit relativement complexe autour de la cavité, cette hypothèse représente relativement bien la réalité quand le revêtement est lisse et que les moments supportés sont négligeables (J.H. ATKINSON, D.M. POTTS, 1977). Contrairement au cas du problème $(\mathrm{Pb} \mathrm{I})$ on s'intéresse par conséquent ici à un ouvrage très flexible.

\section{CAS D'UN TERRAIN ARGILEUX}

La stabilité d'un tunriel creusé en terrain purement cohérent (critère de TRESCA) a fait l'objet de nombreuses études. En général le problème d'un sol non pesant $(\gamma=0)$ et celui d'un sol pesant sont considérés séparément.

Le premier cas constitue une bonne représentation d'un tunnel profond (l'effet local de la pesanteur peut être négligé). Pour un tunnel creusé à faible profondeur en revanche, il est nécessaire de prendre en compte directement le poids des terrains dans l'analyse. L'extension des résultats obtenus pour un sol non pesant au cas $\gamma>0$ nécessite souvent des approximations, qui d'un point de vue purement théorique tendent à modifier le problème étudié.

Pour ce qui est de l'approche statique, il est en fait possible de combiner les deux calculs $(\gamma=0$ et $\gamma>0)$ et d'aboutir à des résultats rigoureux et valables dans le cas général $(\gamma>0)$.

Les champs de contraintes statiquement admissibles et les mécanismes utilisés dans les deux paragraphes qui suivent ont été proposés par E.H. DAVIS, M.J. GUNN, R.J. MAIR, H.N. SENEVIRATNE (1980). Les calculs qui en découlent permettent d'isoler le rapport $\frac{\sigma_{s}-\sigma_{T}}{C_{v}}$. Il est donc intéressant de réécrire les paramètres de chargement $\frac{\gamma_{\mathrm{D}}}{\mathrm{C}_{\mathrm{u}}}, \frac{\sigma_{\mathrm{s}}-\sigma_{\mathrm{T}}}{\mathrm{C}_{\mathrm{u}}}, \frac{\sigma_{\mathrm{s}}+\sigma_{\mathrm{T}}}{\mathrm{C}_{\mathrm{u}}}$. En réalité $\frac{\sigma_{s}+\sigma_{T}}{C_{u}}$ est absent de toutes les relations établies, ce qui signifie que l'ensemble des chargements potentiellement supportables peut être représenté dans le plan $\left(\frac{\gamma_{D}}{C_{u}}, \frac{\sigma_{s}-\sigma_{T}}{C_{u}}\right)$. Ceci permet également d'établir le lien avec le rapport :

$$
N=\frac{\gamma_{H}+\sigma_{5}-\sigma_{T}}{C_{u}}
$$

utilisé dans la pratique pour caractériser la stabilité du front de taille d'un tunnel en milieu argileux. Comme on le voit à la figure (3.3.1) il suffit pour cela de tracer une droite déduite de l'axe $\gamma \mathrm{D} / \mathrm{C}_{\mathrm{u}}$ par une rotation d'angle $\alpha_{c}=\operatorname{Arctg}\left[\frac{1}{\mathrm{C} / \mathrm{D}+1 / 2}\right]$ et le rapport $\mathrm{N}$ est alors donné directement sur cette droite en multiples de $k_{c}=\sqrt{\left(\frac{C}{D}+\frac{1}{2}\right)^{2}+1}$. L'angle $\alpha_{c}$ comme le rapport de proportionnalité $k_{c}$ ne dépendent que des propriétés géométriques du tunnel.

\subsection{Bornes inférieures}

Le champ de contrainte de la figure (3.1.1), qui caractérise la * rupture locale » dans l'étude E.H. DAVIS et

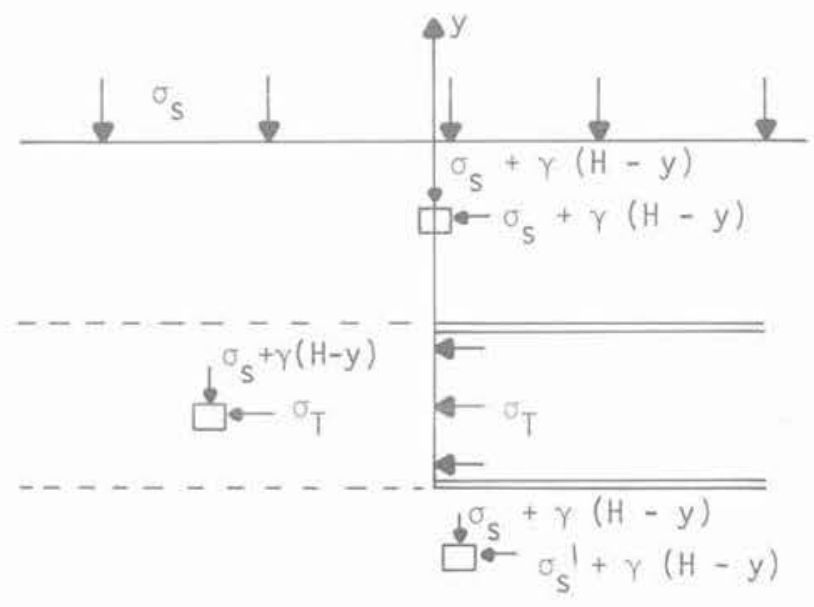

Fig. 3.1.1. - Borne inferieure dans le cas d'un sol pesant. Fig. 3.1.1. - Lower bound solution accounting for soil weight. 
al. (1980) permet d'obtenir une borne inférieure des chargements potentiellement supportables dans le cas général $(\gamma>0)$.

Ce champ de contrainte est bien statiquement admissible et la vérification du critère de résistance conduit à la relation :

$$
\begin{gathered}
\operatorname{Max}\left[\left|\sigma_{\mathrm{s}}+\gamma(\mathrm{H}-\mathrm{y})-\sigma_{\mathrm{T}}\right|\right] \leqslant 2 \mathrm{C}_{\mathrm{u}} \\
-\mathrm{D} / 2 \leqslant \mathrm{y} \leqslant \mathrm{D} / 2
\end{gathered}
$$

soit encore :

$$
\begin{array}{r}
N+\frac{1}{2} \frac{\gamma_{D}}{C_{u}} \leqslant 2 \\
-N+\frac{1}{2} \frac{\gamma_{D}}{C_{u}} \leqslant 2
\end{array}
$$

Dans le plan des chargements, ces relations peuvent chacune être représentées, lorsqu'il y a égalité par une droite - $A B$ et $A^{\prime} B$ respectivement sur la figure (3.3.1) - ce qui signifie que tout point situé à l'intérieur du triangle $A B A^{\prime}$ correspond à un chargement potentiellement supportable.

Les solutions établies dans le cas $\gamma=0$ associées à la propriété de convexité du domaine (P) des chargements potentiellement supportables vont maintenant être mises à profit pour élargir le triangle $A B A^{\prime}$ et obtenir une approche par l'intérieur plus réaliste du domaine $(\mathrm{P})$.

Les champs de contraintes représentés sur les figures

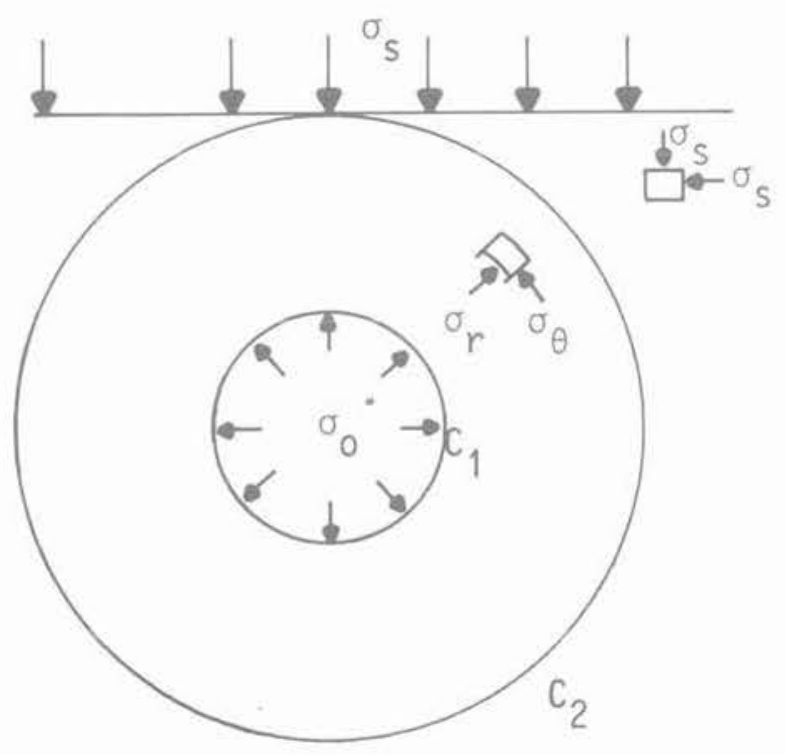

Coupe A-A
(3.1.2) et (3.1.3) fournissent chacun un chargement potentiellement supportable en sol non pesant. Le premier, à symétrie cylindrique autour de l'axe du tunnel

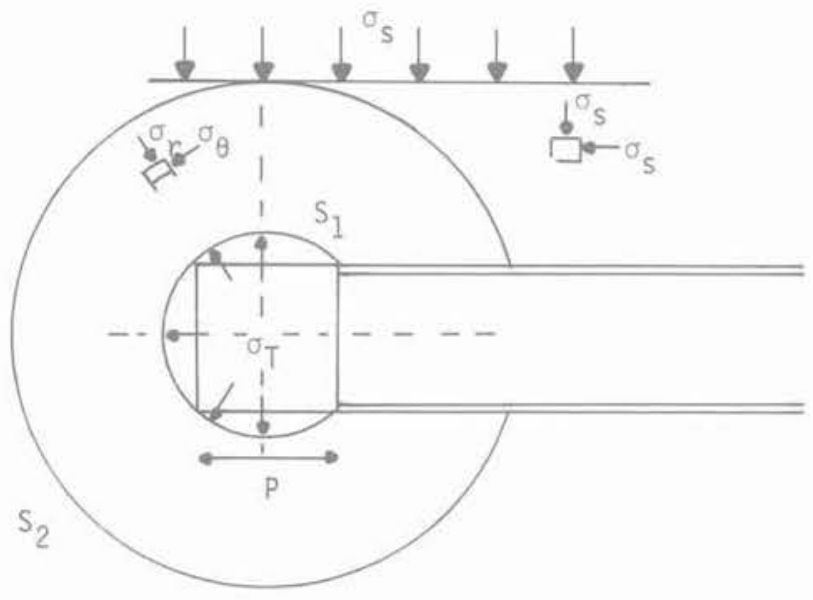

Fig. 3.1.3. - Champ à symétrie sphérique. Le critère est atteint entre les sphères $S_{1}$ et $S_{2}$.

Fig. 3.7.3. - Case of a spherically symetric stress field

Soil located berween spheres $S_{1}$ and $S_{2}$ is in a state plastic yield.

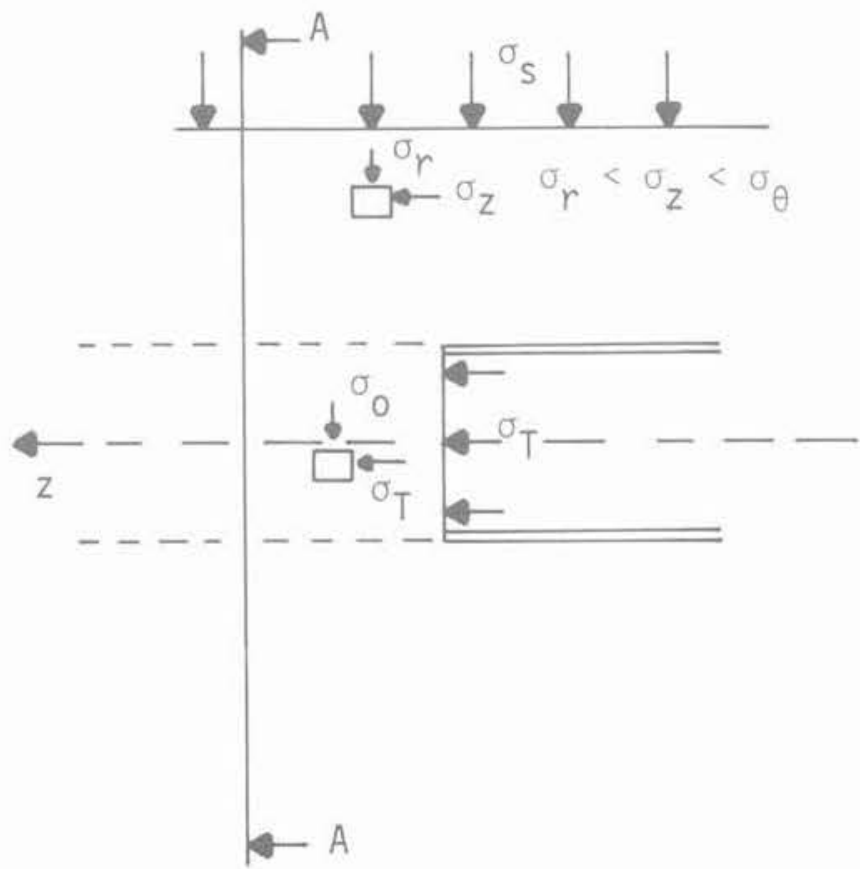

Fig. 3.1.2. - Champ à symétrie cylindrique. Le critère est atteint entre les cylindres $C_{1}$ et $C_{2}$ Fig. 3.1.2. - Case of a radially symetric stress field. Soil located between cylinders $C_{1}$ and $C_{2}$ is in a state of plastic yield. 
conduit à une borne inférieure qui se met sous la forme :

$$
\left[\frac{\sigma_{\mathrm{s}}-\sigma_{\mathrm{T}}}{\mathrm{C}_{\mathrm{u}}}\right]^{-}=2+2 \ln \left(2 \frac{\mathrm{C}}{\mathrm{D}}+1\right)
$$

Le second, à symétrie sphérique au niveau du front de taille permet d'écrire :

$$
\left[\frac{\sigma_{\mathrm{s}}-\sigma_{\mathrm{T}}}{\mathrm{C}_{\mathrm{u}}}\right]^{-}=4 \ln \left(2 \frac{\mathrm{C}}{\mathrm{D}}+1\right)
$$

L'examen des relations (3.1.3) et (3.1.4) montre que le champ à symétrie cylindrique fournit un \& meilleur minorant " lorsque C/D est inférieur à 0.86 et que le champ à symétrie sphérique fournit un « meilleur minorant " pour $C / D \geqslant 0.86$. Ce "meilleur minorant » définit un point du domaine, représenté par le point D sur la figure $(3,3.1)$

Les relations (3.1.3) et (3.1.4) sont basées sur l'hypothèse implicite que la contrainte orthoradiale $\sigma_{\theta}$ est plus forte que la contrainte radiale $\sigma$, dans la région où le critère de résistance du sol est atteint. Ceci correspond à un risque de rupture par effondrement de la galerie. Si maintenant on étudie le problème d'une rupture par explosion $\left(\sigma_{T}>\sigma_{\theta}\right)$, les mêmes calculs permettent de mettre en évidence un point D', symétrique de D par rapport à l'axe des $\gamma \mathrm{D} / \mathrm{C}_{\mathrm{u}}$ sur la figure $(3.3 .1)$ et qui lui aussi appartient à $(\mathrm{P})$.

Ainsi les points B, D et D' sont dans (P). La convexité de cet ensemble permet d'affirmer que le triangle DBD' est par conséquent aussi dans (P). Le triangle DBD' constitue une meilleure approche par l'intérieur du domaine des chargements potentiellement supportables, et ceci pour toute valeur du poids volumique du sol $(\gamma=0$ ou $\gamma>0)$.

\subsection{Bornes supérieures}

Dans cette partie les résultats de E.H. DAVIS et al. (1980) ont été repris sans modifications et représentés sur le diagramme de la figure (3.3.1). En particulier l'équivalence entre sol non pesant et sol pesant a été admise pour le mécanisme d'effondrement représenté à la figure (3.2.1). Toutefois, les calculs développés à l'annexe I montrent que dans le cas plus simple d'une longue taille dans une mine ( $\mathrm{Pb}$ II), le résultat obtenu pour $\gamma=0$ est bien généralisable au cas $\gamma>0$, à condition de remplacer $\frac{\sigma_{s}-\sigma_{T}}{C_{u}}$ par $N=\frac{\sigma_{s}-\sigma_{T}+\gamma_{H}}{C_{u}}$. Pour le problème tridimensionnel $(\mathrm{Pb} \mathrm{I})$ la borne supérieure $\left[\frac{\sigma_{s}-\sigma_{T}+\gamma_{H}}{C_{u}}\right]+=[N]+$ est aussi une fonction de $\frac{\mathrm{C}}{\mathrm{D}}$, qui est donnée à la figure $(3.2 .2)$.

Dans le plan $\left(\frac{\gamma_{D}}{C_{u}}, \frac{\sigma_{s}-\sigma_{T}}{C_{u}}\right)$ (figure $(3.3 .1$.$) ) cette$ borne supérieure est représentée par une droite $\mathrm{N}=$ constante, c'est-à-dire perpendiculaire à l'axe des $\mathrm{N}$ (la valeur de C/D a été choisie égale à 2 sur la figure $(3.3 .1)$; dans ce cas $\left.[\mathrm{N}]^{+}=12.23\right)$.

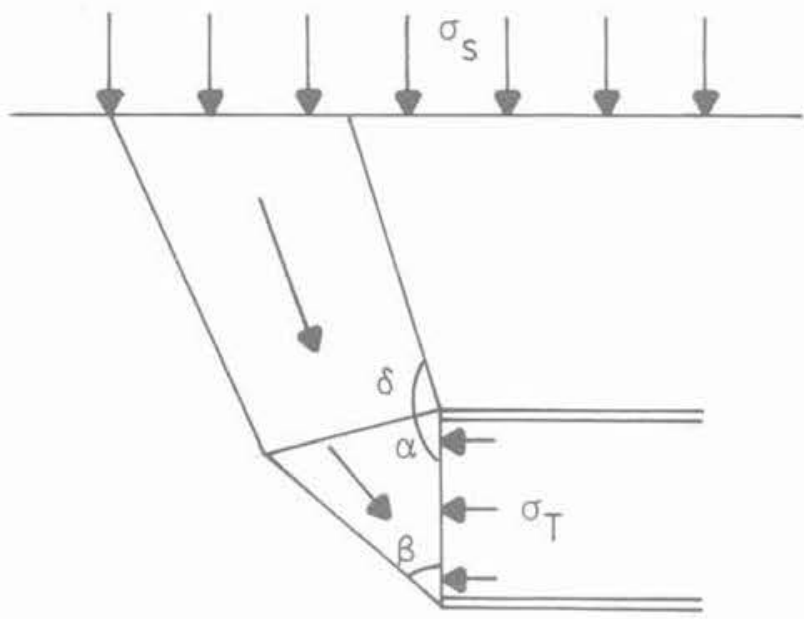

Fig. 3.2.1. - Mécanisme de rupture par effondrement. Fig. 3.2.1. - Upper bound mechanism for a global collapse into the unnel.

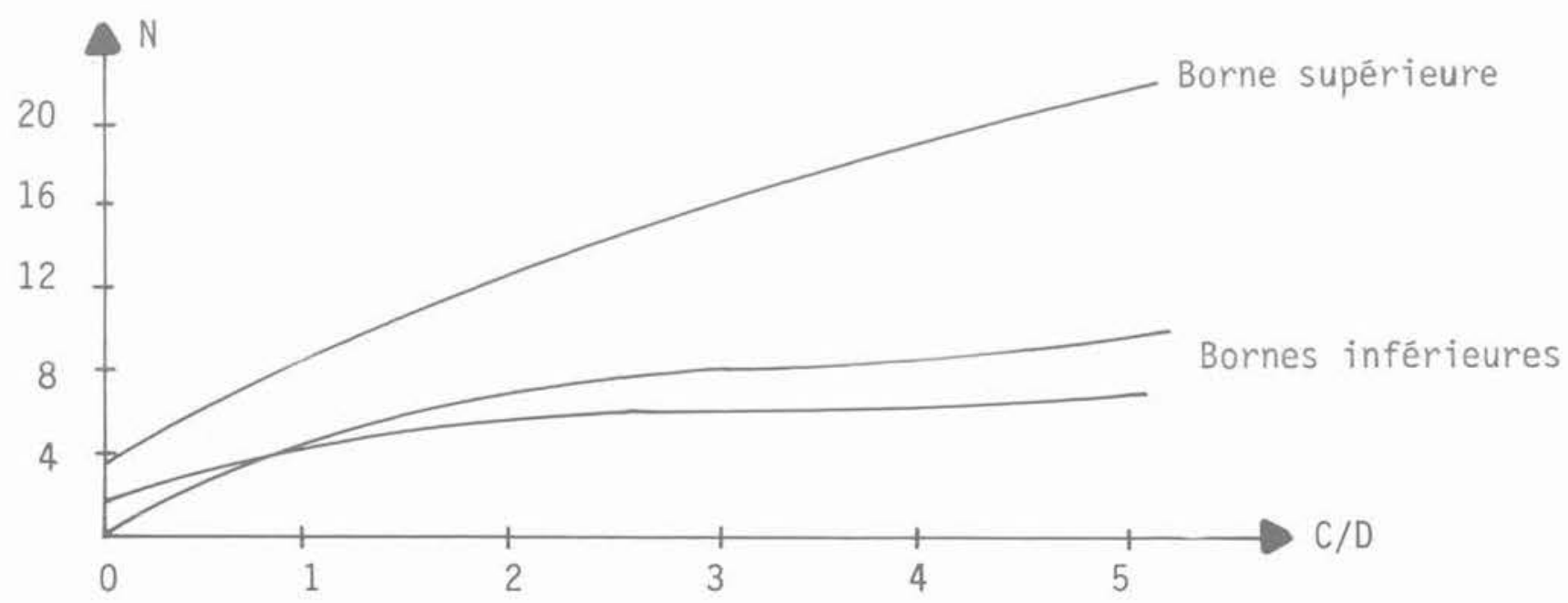

Fig. 3.2.2. - Borne supérieure fournie par le mécanisme de la figure (3.2.1.).

Fig. 3.2.2 - Upper bound solution for the mechanism shown on figure 3.2.1. 
Si on considère maintenant le même mécanisme, mais dans le cas d'une rupture par explosion, l'inversion du sens des vitesses sur la figure (3.2.1) conduit à rempla$\operatorname{cer} \sigma_{s}-\sigma_{T}+\gamma\left(C+\frac{D}{2}\right)$ par $\sigma_{T}-\sigma_{s}-\gamma\left(C+\frac{D}{2}\right)$

dans l'expression de la puissance des efforts extérieurs. On obtient ainsi une autre borne supérieure de l'ensemble des chargements potentiellement supportables en traçant la droite $\mathrm{N}=-12.23$ (quand $C / D=2)$ symétrique de la précédente par rapport à laxe $\mathrm{N}=0$.

Par conséquent, le domaine $(\mathrm{P})$ doit être compris entre les droites EF et E'F' de la figure (3.3.1). L'optimisation du mécanisme de "rupture locale * représenté à la figure (3.2.3) permet de boucler l'approche de (P) par l'extérieur.

La borne supérieure s'écrit en effet (9) :

$$
\left[\frac{\gamma_{D}}{C_{u}}\right]^{+}=10.96
$$

et correspond, sur la figure (3.3.1) à la droite FF'.

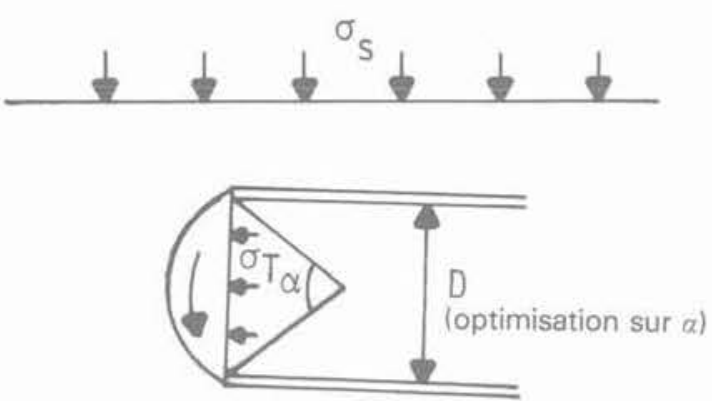

Fig. 3.2.3, - Mécanisme de " rupture locale ". Fig. 3.2.3. - Local collapse mechanism.

\subsection{Encadrement des chargements potentiellement supportables}

Les résultats précédents peuvent être rassemblés sur un diagramme $\left(\frac{\gamma_{D}}{C_{u}}, \frac{\sigma_{s}-\sigma_{T}}{C_{u}}\right)$. Ce diagramme est entièrement déterminé dès que $\mathrm{C} / \mathrm{D}$ est connu. La figure (3.3.1) correspond au cas où $C / D=2$. Les paramètres $\gamma$, D et $C_{u}$ étant tous trois positifs, l'ensemble $(\mathrm{P})$ des chargements potentiellement supportables est nécessairement contenu dans le demi-plan $\frac{\gamma_{D}}{C_{u}} \geqslant 0$. Sa frontière est comprise entre le triangle DBD' et le parallélogramme EFF'E'.

Il ressort de cette analyse qu'il est possible de tirer parti de résultats obtenus dans les conditions simples d'un sol non pesant pour améliorer l'approche par l'intérieur du domaine $(P)$ dans le cas général $(\gamma \geqslant 0)$. Néanmoins, comme on peut le voir sur la figure (3.3.1) la marge est encore importante entre borne inférieure et borne supérieure et l'examen d'autres champs de contraintes et d'autres mécanismes devrait permettre de resserrer l'encadrement des chargements potentiellement supportables.

\section{CAS D'UN TERRAIN FROTTANT-COHÉRENT}

Lorsque le massif excavé est frottant et cohérent, ses propriétés de résistance sont décrites par un critère de MOHR-COULOMB et le problème devient plus complexe. Contrairement au cas d'une argile, on dispose ici de peu d'éléments pour caractériser de manière rigoureuse sur le plan analytique, la stabilité du front de taille du tunnel.

Dans la présente étude, seule l'approche par l'intérieur a été considérée. Quelques bornes inférieures ont pu être mises en évidence, en s'inspirant, pour le choix des champs de contrainte admissibles, des travaux concernant les matériaux, purement cohérents. Les résultats sont bien sûr moins simples que dans ce dernier cas et il est nécessaire de prendre effectivement en compte trois paramètres de chargement distincts :

$$
\frac{\sigma_{s}}{\sigma_{c}}, \frac{\sigma_{T}}{\sigma_{c}}, \frac{\gamma_{D}}{\sigma_{c}} \text {. }
$$

\subsection{Borne inférieure dans le cas général}

Le champ de contrainte décrit à la figure (3.1.1) peut être repris dans le cas d'un matériau obéissant à un critère de MOHR-COULOMB. Les calculs sont développés à l'annexe Il. Ils montrent qu'un champ statiquement et plastiquement admissible doit vérifier les deux relations suivantes :

$$
\begin{gathered}
\frac{\sigma_{s}}{\sigma_{c}}-K_{p} \frac{\sigma_{\mathrm{T}}}{\sigma_{\mathrm{c}}}+\frac{\gamma_{\mathrm{D}}}{\sigma_{\mathrm{c}}}\left(\frac{\mathrm{C}}{\mathrm{D}}+1\right) \leqslant 1 \\
\frac{\sigma_{\mathrm{T}}}{\sigma_{\mathrm{c}}}-\mathrm{K}_{\mathrm{p}} \frac{\sigma_{\mathrm{s}}}{\sigma_{\mathrm{c}}}-\mathrm{K}_{\mathrm{p}} \frac{\gamma_{\mathrm{D}}}{\sigma_{\mathrm{c}}} \frac{\mathrm{C}}{\mathrm{D}} \leqslant 1 \\
\text { où } \mathrm{K}_{\mathrm{p}}=\frac{1+\sin \varphi^{\prime}}{1-\sin \varphi^{\prime}}
\end{gathered}
$$

Autrement dit, on peut limiter le domaine des chargements potentiellement supportables dans l'espace $\left(\frac{\sigma_{s}}{\sigma_{c}}, \frac{\sigma_{\mathrm{T}}}{\sigma_{\mathrm{c}}}, \frac{\gamma_{\mathrm{D}}}{\sigma_{\mathrm{c}}}\right)$ par deux plans d'équations :

$$
\begin{gathered}
\frac{\sigma_{\mathrm{s}}}{\sigma_{\mathrm{c}}}-\mathrm{K}_{\mathrm{p}} \frac{\sigma_{\mathrm{T}}}{\sigma_{\mathrm{c}}}+\frac{\gamma_{\mathrm{D}}}{\sigma_{\mathrm{c}}}\left(\frac{\mathrm{C}}{\mathrm{D}}+1\right)=1 \\
\frac{\sigma_{\mathrm{T}}}{\sigma_{\mathrm{c}}}-\mathrm{K}_{\mathrm{p}} \frac{\sigma_{\mathrm{s}}}{\sigma_{\mathrm{c}}}-K_{\mathrm{p}} \frac{\gamma_{\mathrm{D}}}{\sigma_{\mathrm{c}}} \frac{\mathrm{C}}{\mathrm{D}}=1
\end{gathered}
$$

\subsection{Bornes inférieures en sol non pesant}

Comme dans le cas d'un terrain purement cohérent, la considération d'un champ de contrainte à symétrie cylindrique autour de l'axe du tunnel et d'un champ à symétrie sphérique au niveau du front de taille permet d'aboutir à deux nouvelles bornes inférieures en sol non pesant.

Le champ de contrainte de la figure (3.1.2) est analysé 


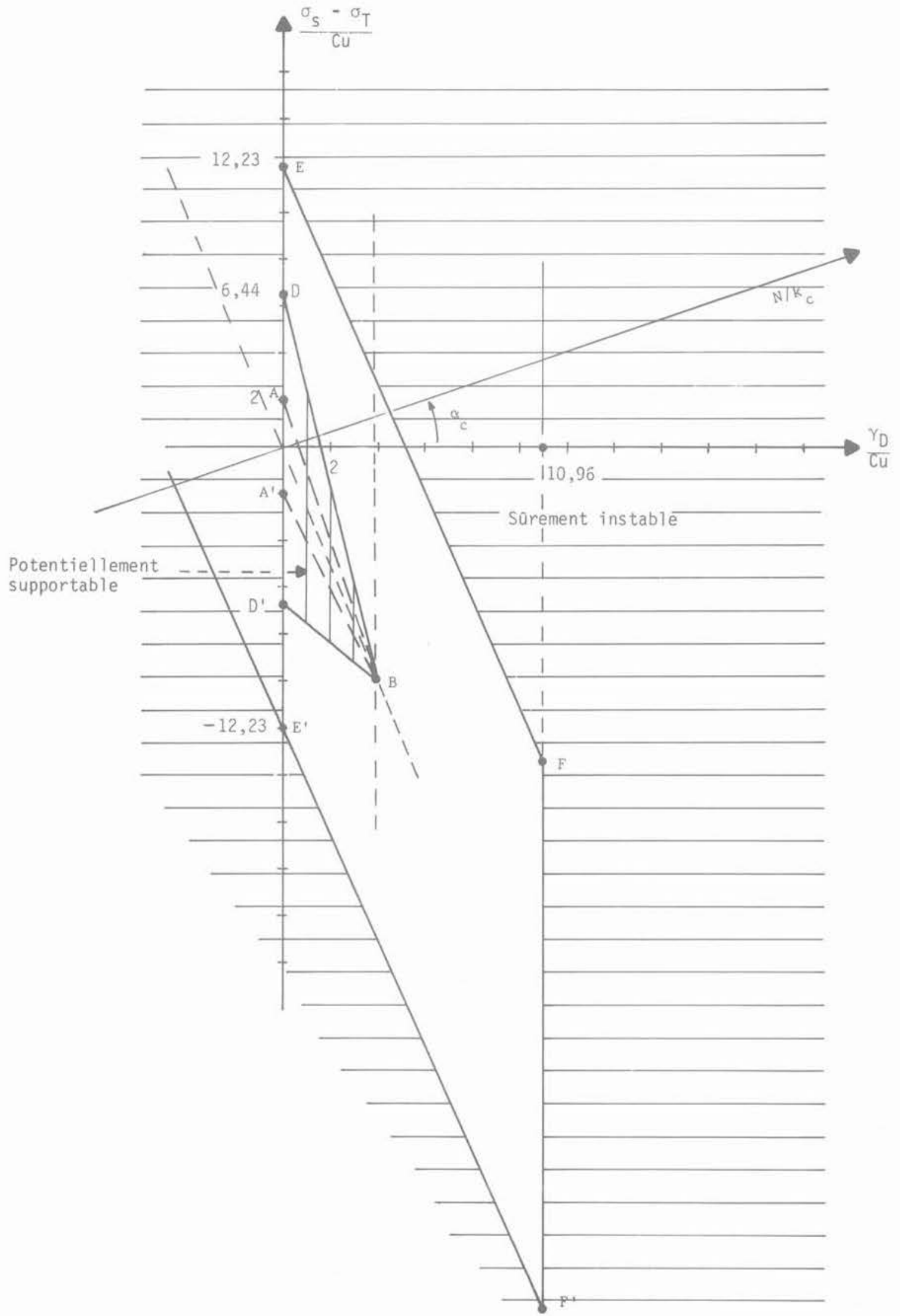

Fig. 3.3.1. - Encadrement du domaine (P).

Fig. 3.3.1. - Bracketting for domain (P). 
à l'annexe III. Le résultat peut se mettre sous la forme :

$$
\begin{aligned}
\frac{1}{K_{p}}\left(2 \frac{C}{D}+1\right)\left(\frac{1}{K_{p}}-1\right) & \leqslant \frac{\left(K_{p}-1\right) \frac{\sigma_{s}}{\sigma_{c}}+1}{\left(K_{p}-1\right) \frac{\sigma_{T}}{\sigma_{c}}+1} \\
& \left.\leqslant K_{p}\left(2 \frac{C}{D}+1\right) K_{p}-1\right)
\end{aligned}
$$

La relation (4.2.1) correspond bien sûr à un critère de MOHR-COULOMB vrai $\left(\mathrm{K}_{\mathrm{p}}>1\right)$. Comme on peut le voir à l'annexe III, on retrouve exactement la relation (3.1.3) lorsque $\mathrm{K}_{\mathrm{p}}=1$ (sol purement cohérent).

En ce qui concerne le champ à symétrie sphérique (figure 3.1.3) les calculs de l'annexe IV montrent que le résultat pourrait être déduit d'une généralisation des travaux récents de H.B. MUHLHAUS (1985). Ce dernier considère la longueur limite $\mathrm{P}$ de tunnel non soutenu au front de taille pour un champ à symétrie sphérique (figure 4.2.1). La même approche est utilisée à l'annexe IV, mais une pression interne $\sigma_{\mathrm{T}}$ est ajoutée au système de chargement. $\mathrm{Si}$ on se limite au cas $\mathrm{P} / \mathrm{D}=0$ et qu'on s'intéresse aux pressions limites appliquées au tunnel, le résultat s'écrit :

$$
\begin{gathered}
\left(2 \frac{C}{D}+1\right)^{2}\left(\frac{1}{K_{p}}-1\right) \leqslant \frac{\left(K_{p}-1\right) \frac{\sigma_{s}}{\sigma_{c}}+1}{\left(K_{p}-1\right) \frac{\sigma_{T}}{\sigma_{c}}+1} \\
\leqslant\left(2 \frac{C}{D}+1\right)^{2\left(K_{p}-1\right)}
\end{gathered}
$$

Ici aussi la condition $\mathrm{K}_{\mathrm{p}}=1$ conduirait exactement à la relation (3.1.4) établie en sol purement cohérent.

On peut constater que dans les deux cas (relations 4.2.1 et 4.2.2), le résultat s'écrit sous la forme d'une

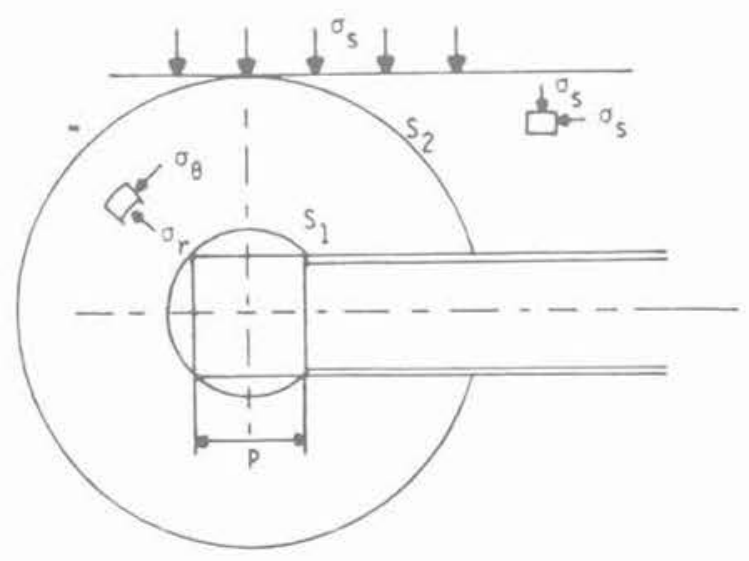

Fig. 4.2.1. - Champ de contrainte considéré par H.B. MUHLAUS (1985).

Fig. 4.2.1. - Stress field used by H.B. MUHLAUS (1985). double inégalité. Ceci résulte du fait que deux types de rupture d'ensemble peuvent se produire au front de taille. La pression $\sigma_{\mathrm{T}}$ doit être suffisante pour retenir le terrain, mais si elle devient trop forte, il y a explosion de la galerie.

\subsection{Discussion}

Les champs de contraintes considérés dans l'approche par l'intérieur pour le cas simple d'un sol purement cohérent peuvent également servir à déterminer des bornes inférieures dans un terrain obéissant à un critère de MOHR-COULOMB. Ici encore l'étude est conduite séparément en sol pesant d'une part, en sol non pesant d'autre part et le même principe de convexité pourrait être utilisé pour optimiser l'approche par l'intérieur des chargements potentiellement supportables à partir des résultats obtenus pour $\gamma=0$. Une telle opération doit

bien sûr s'effectuer dans l'espace des chargements $\left.\frac{\sigma_{5}}{\sigma_{\mathrm{c}}}, \frac{\sigma_{\mathrm{T}}}{\sigma_{\mathrm{c}}}, \frac{\gamma_{\mathrm{D}}}{\sigma_{\mathrm{c}}}\right)$ et la visualisation est plus délicate que pour le cas d'un sol non frottant où le problème peut être analysé dans le plan $\left(\frac{\gamma_{\mathrm{D}}}{\mathrm{C}_{\mathrm{u}}}, \frac{\sigma_{\mathrm{s}}-\sigma_{\mathrm{T}}}{\mathrm{C}_{\mathrm{u}}}\right)$.

Les conclusions de la présente étude sont néanmoins relativement encourageantes et montrent que pour un terrain obéissant à un critère de MOHR-COULOMB, le calcul à la rupture peut encore fournir des résultats intéressants quant à la stabilité du front de taille d'un tunnel. En particulier, dans le cas $\gamma=0$, les relations (4.2.1) et (4.2.2) fournissent une meilleure borne infé. rieure pour un sol non cohérent que le critère proposé par J.H. ATKINSON et D.M. POTTS :

$$
\frac{\sigma_{5}}{\sigma_{T}} \leqslant\left(2 \frac{C}{D}+1\right)^{k_{p}-1}
$$

La relation (4.2.3) résulte en fait d'un calcul bidimensionnel (problème $\mathrm{Pb}$ III) ; la généralisation au cas étudié ici est basée sur le résultat expérimental que la section courante d'un tunnel (modélisée par Pb III) est plus sensible à l'effondrement que le front de taille (modélisé par $\mathrm{Pb}$ I). Il est donc naturel que la relation (4.2.1) aboutisse à un meilleur résultat ; considérer le problème ( $\mathrm{Pb}$ III) revient en effet à supposer que le champ de contrainte représenté à la figure (3.1.2) est égal à $\sigma_{\mathrm{T}}$ au lieu de $\sigma_{0}$ à l'intérieur du cylindre $C_{1}$; par conséquent les capacités de résistance du terrain sont moins bien utilisées (pour aboutir à la relation (4.2.1), $\sigma_{0}$ est ajusté pour que le critère de résistance soit exactement atteint dans $C_{1}$ ).

\section{CONCLUSION}

Le calcul à la rupture apparaît comme un outil particulièrement efficace pour obtenir des informations sur la stabilité du front de taille d'un tunnel.

Dans le cas d'un matériau purement cohérent (critère de TRESCA), il est possible de représenter les résultats dans le plan $\left(\frac{\gamma_{\mathrm{D}}}{\mathrm{C}_{\mathrm{u}}}, \frac{\sigma_{\mathrm{s}}-\sigma_{\mathrm{T}}}{\mathrm{C}_{\mathrm{u}}}\right)$. 
On aboutit alors à un encadrement des chargements * potentiellement supportables * qui peut être rattaché aux critères utilisés dans la pratique, basés sur le rapport

$$
N=\frac{\sigma_{s}+\gamma_{H}-\sigma_{T}}{C_{u}}
$$

La présente étude a montré qu'un travail analogue est réalisable pour des terrains frottants-cohérents (critère de MOHR-COULOMB), du moins pour ce qui est de l'approche par l'intérieur. La principale difficulté provient du fait que trois paramètres de chargements doivent alors être considérés $\frac{\sigma_{s}}{\sigma_{c}}, \frac{\sigma_{T}}{\sigma_{c}}, \frac{\gamma_{D}}{\sigma_{c}}$, et la visualisation des résultats est par conséquent plus délicate.

D'une manière générale, les auteurs qui se sont intéressés au problème de la stabilité du front de taille d'un tunnel s'accordent pour signaler une bonne correspondance entre résultats expérimentaux et bornes inférieures mises en évidence par le calcul à la rupture ou l'analyse limite. Néanmoins l'encadrement des chargements "potentiellement supportables" en terrain purement cohérent est encore relativement grossier : en ce qui concerne les sols frottants-cohérents, l'approche par l'extérieur n'a pas vraiment été développée. De nouveaux champs de contraintes et d'autres mécanismes, inspirés de l'observation expérimentale devraient donc être envisagés de manière à améliorer les résultats actuellement disponibles.

\section{ANNEXE}

Calcul d'une borne supérieure pour $\mathrm{Pb}$ II dans le cas où $\gamma>\mathrm{C}$

On se place dans l'hypothèse :

$$
\begin{gathered}
\operatorname{tg} \alpha=2 \sqrt{\frac{C}{D}+\frac{1}{4}} \\
\delta=\frac{\pi}{2}
\end{gathered}
$$

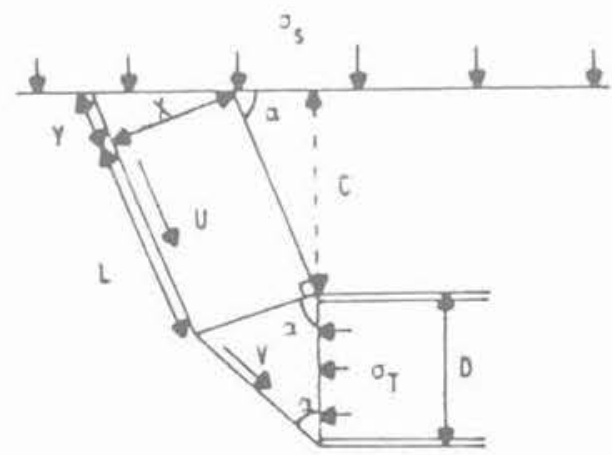

Fig. 1 (annexe I).
De plus, pour un matériau de TRESCA, la nécessité d'avoir une puissance dissipable finie conduit à considérer qu'il y a glissement des deux blocs sans décollement, c'est-à-dire que la discontinuité de vitesse entre les deux blocs est parallèle à la surface de contact (figure 2)

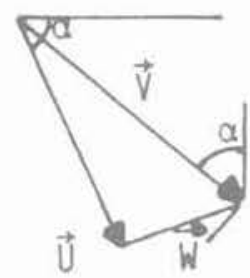

Fig. 2 (annexe I).

Le calcul des paramètres introduits sur les figures 1 et 2 donne :

$$
\begin{aligned}
& \mathrm{U}=\mathrm{V} \cos \left(\frac{\pi}{2}-2 \alpha\right) \\
& \mathrm{W}=\mathrm{V} \cos (\pi-2 \alpha)
\end{aligned}
$$

donc :

$\mathrm{U}=\mathrm{V} \sin 2 \alpha$

$$
\mathrm{UX}=\mathrm{V} \cos \frac{1}{2}(\pi-2 \alpha) \mathrm{D}
$$

$W=-V \cos 2 \alpha$

(conservation du volume déplacé par le mouvement des deux blocs), donc :

$$
X=\frac{D}{2 \cos \alpha}
$$

Enfin :

$$
\begin{aligned}
& \operatorname{tg} \alpha=\frac{X}{Y} \\
& \sin \alpha=\frac{C}{L}
\end{aligned}
$$

donc :

$$
\begin{aligned}
& Y=\frac{D}{2 \sin \alpha} \\
& L=\frac{C}{\sin \alpha}
\end{aligned}
$$

La puissance $\mathrm{P}_{e}$ des efforts extérieurs dans ce mécanisme a pour expression :

$$
\begin{aligned}
& \mathrm{P}_{\mathrm{e}}=\left(\sigma_{\mathrm{s}}-\sigma_{\mathrm{T}}\right) \mathrm{VD} \sin \alpha+ \gamma \mathrm{U} \sin \alpha\left(\mathrm{XL}+\frac{1}{2} \mathrm{XY}\right) \\
&+\gamma \mathrm{V} \cos \alpha \frac{1}{2} \mathrm{DX} \sin \alpha \\
&=\left(\sigma_{\mathrm{s}}-\sigma_{\mathrm{T}}\right) \mathrm{VD} \sin \alpha+\gamma \mathrm{V} \sin 2 \alpha \sin \alpha\left(\frac{\mathrm{D}}{2 \cos \alpha}\right.
\end{aligned}
$$


$\left.\frac{C}{\sin \alpha}+\frac{1}{2} \frac{D}{2 \cos \alpha} \frac{D}{2 \sin \alpha}\right)+\gamma V \cos \alpha \frac{1}{2} D$ $\frac{\mathrm{D}}{2 \cos \alpha} \sin \alpha$

soit :

$P_{e}=\left(\sigma_{s}-\sigma_{\tau}+\gamma\left(C+\frac{D}{2}\right)\right) V D \sin \alpha$

La puissance dissipable $\mathrm{P}(\mathrm{v})$ s'écrit :

$P(\underline{v})=C_{u}(2 L+Y) U+C_{u} Y W+C_{u} X V$

$=C_{u}\left(2 \frac{C}{\sin \alpha}+\frac{D}{2 \sin \alpha}\right) V \sin 2 \alpha+C_{u} \frac{D}{2 \cos \alpha}$

$$
(-\mathrm{V} \cos 2 \alpha)+C_{u} \frac{D}{2 \cos \alpha} V
$$

$=4 C_{u} V \cos \alpha\left(C+\frac{D}{4}+\frac{D}{4} \operatorname{tg}^{2} \alpha\right)$

Compte tenu de $\operatorname{tg} \alpha=2 \sqrt{\frac{C}{D}+\frac{1}{4}}$. On en déduit :

$P(\underline{v})=4 C_{u} V D \cos \alpha 2\left(\frac{C}{D}+\frac{1}{4}\right)$

L'application du théorème de la borne supérieure conduit à dire que le chargement ne sera pas supportable si :

$$
\begin{gathered}
\left(\sigma_{\mathrm{s}}-\sigma_{\mathrm{T}}+\gamma\left(\mathrm{C}+\frac{\mathrm{D}}{2}\right)\right) \mathrm{VD} \\
\sin \alpha \geqslant 4 \mathrm{C}_{\mathrm{u}} \vee \mathrm{D} \cos \alpha 2\left(\frac{\mathrm{C}}{\mathrm{D}}+\frac{1}{4}\right)
\end{gathered}
$$

ce qui donne comme majorant des chargements potentiellement supportables:

$$
\left[\sigma_{\mathrm{s}}-\sigma_{\mathrm{T}}+\gamma\left(\mathrm{C}+\frac{\mathrm{D}}{2}\right)\right]^{+}=4 \mathrm{C}_{\mathrm{u}} \frac{2\left(\frac{\mathrm{C}}{\mathrm{D}}+\frac{1}{4}\right)}{\operatorname{tg} \alpha}
$$

c'est-à-dire :

$$
\left[\frac{\sigma_{s}-\sigma_{\mathrm{T}}+\gamma\left(\mathrm{C}+\frac{\mathrm{D}}{2}\right.}{\mathrm{C}_{u}}\right]+=4 \sqrt{\frac{\mathrm{C}}{\mathrm{D}}+\frac{1}{4}}
$$

le résultat est le même que pour $\gamma=0$, à condition de remplacer $\frac{\sigma_{s}-\sigma_{T}}{C_{u}} \operatorname{par} N=\frac{\sigma_{s}-\sigma_{T}+\gamma(C+D / 2)}{C_{u}}$

\section{ANNEXE II}

Recherche d'une borne inférieure pour un sol de MOHR-COULOMB quand $\gamma>0$

On considère le champ de contrainte de la figure 1 .

Un tel champ est statiquement admissible par construction. Il reste à vérifier le critère de résistance du matériau :

$$
\sigma_{1} \leqslant \mathrm{~K}_{\mathrm{p}} \sigma_{3}+\sigma_{\mathrm{c}}
$$

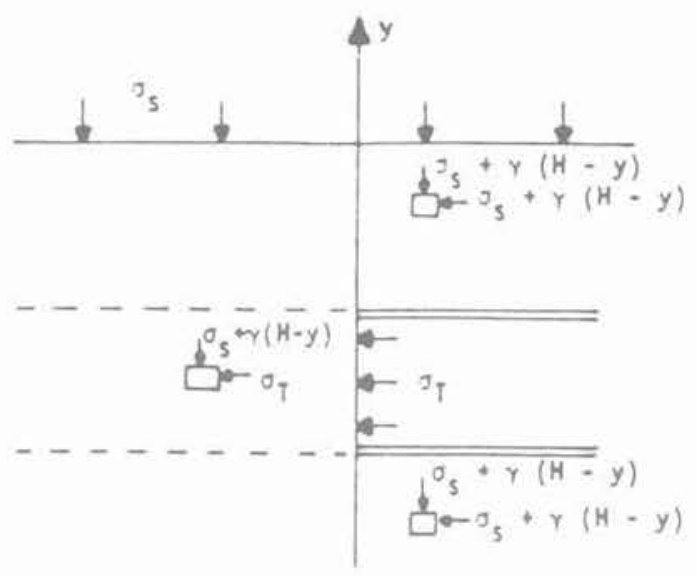

Fig. 1 (annexe II).

Pour cela, on doit distinguer deux cas :

1er cas : $\sigma_{1}=\sigma_{y}$

La relation (1) s'écrit alors :

$$
\begin{aligned}
& \operatorname{Max}\left(\sigma_{\mathrm{s}}+\gamma(\mathrm{H}-\mathrm{y})\right) \leqslant \mathrm{K}_{\mathrm{p}} \sigma_{\mathrm{T}}+\sigma_{c} \\
& -\frac{\mathrm{D}}{2} \leqslant \mathrm{y} \leqslant \frac{\mathrm{D}}{2}
\end{aligned}
$$

c'est-à-dire :

$$
\frac{\sigma_{s}}{\sigma_{c}}-K_{p} \frac{\sigma_{T}}{\sigma_{c}}+\frac{\gamma_{D}}{\sigma_{c}}\left(\frac{C}{D}+1\right) \leqslant 1
$$

$2^{e}$ cas $: \sigma_{1}=\sigma_{\mathrm{T}}$

La relation (1) donne :

$$
\begin{gathered}
\sigma_{\mathrm{T}} \leqslant \mathrm{K}_{\mathrm{p}} \operatorname{Min}\left(\sigma_{3}+\gamma(\mathrm{H}-\mathrm{y})+\sigma_{\mathrm{c}}\right. \\
-\frac{\mathrm{D}}{2} \leqslant \mathrm{y} \leqslant \frac{\mathrm{D}}{2}
\end{gathered}
$$

c'est-à-dire :

$$
\frac{\sigma_{T}}{\sigma_{c}}-K_{p} \frac{\sigma_{s}}{\sigma_{c}}-K_{p} \frac{\gamma_{D}}{\sigma_{c}} \frac{C}{D} \leqslant 1
$$

\section{ANNEXE III}

Recherche d'une borne inférieure pour un sol de MOHR-COULOMB quand $\gamma=0$

On considère le champ de contrainte de la figure 1.

Le champ est uniforme et vaut $\sigma_{\mathrm{s}}$ au-delà du cylindre $\mathrm{C}_{2}$. Il est radial dans le plan $\mathrm{z}=$ cte à l'intérieur du cylindre $C_{1}$ et pour $z>0$. Dans cette même région la contrainte axiale vaut $\sigma_{T}$ et la contrainte radiale $\sigma_{0}$ est choisie de manière à vérifier le critère de résistance du matériau :

$$
\sigma_{1}=\mathrm{K}_{\mathrm{p}} \sigma_{3}+\sigma_{\mathrm{c}}
$$



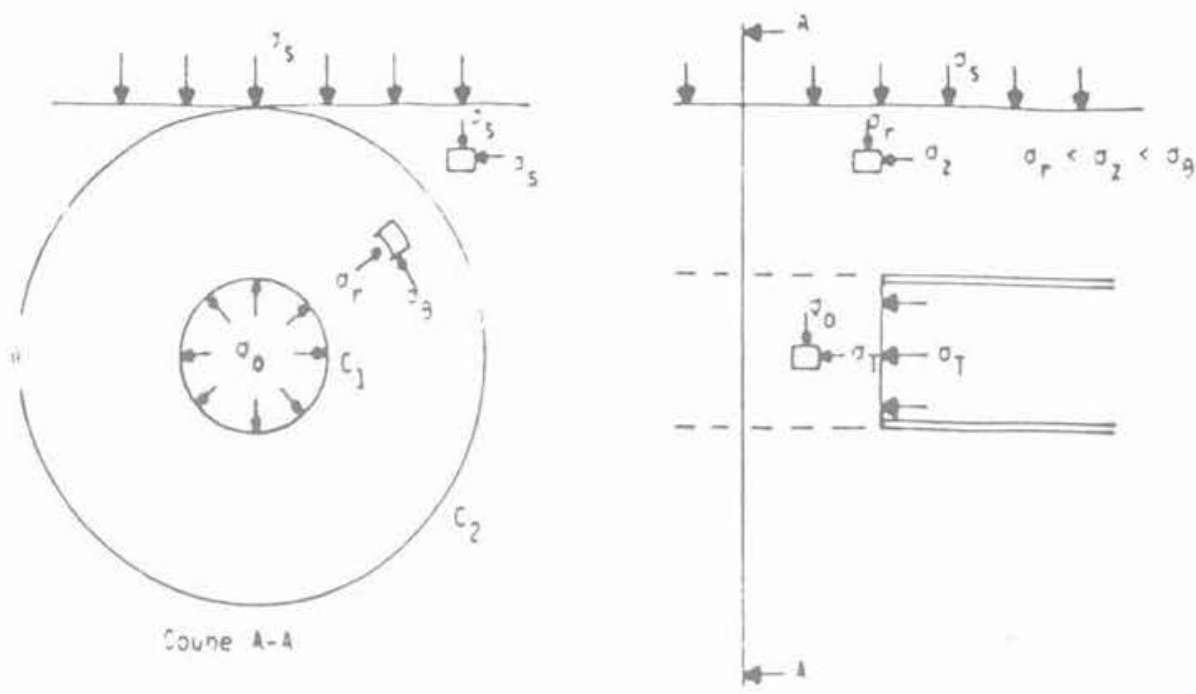

Fig. 1 (annexe III).

Le champ de contrainte entre les deux cylindres est choisi de manière à vérifier les conditions d'équilibre et les conditions aux limites, $\sigma_{\tau}$ étant contrainte intermédiaire et le critère étant atteint (relation (1) vérifiée) :

$$
\begin{aligned}
& \frac{\mathrm{d} \sigma_{\mathrm{r}}}{\mathrm{d}_{\mathrm{f}}}+\frac{\sigma_{\mathrm{r}}-\sigma_{\theta}}{\mathrm{r}}=0 \\
& \sigma_{\mathrm{r}}=\sigma_{\mathrm{s}} \text { pour } \mathrm{r}=\mathrm{H} \\
& \sigma_{\mathrm{t}}=\sigma_{\mathrm{o}} \text { pour } \mathrm{r}=\frac{\mathrm{D}}{2}
\end{aligned}
$$

Deux cas sont à considérer :

1 et cas : $\sigma_{1}=\sigma_{\theta}$

On a alors d'après (1) :

$$
\begin{aligned}
& \sigma_{\mathrm{o}}=\mathrm{K}_{\mathrm{p}} \sigma_{\mathrm{T}}+\sigma_{\mathrm{c}} \\
& \sigma_{\theta}=\mathrm{K}_{\mathrm{p}} \sigma_{\mathrm{t}}+\sigma_{\mathrm{c}}
\end{aligned}
$$

l'équation (2) s'écrit donc :

$$
\frac{\mathrm{d}_{\sigma_{r}}}{\mathrm{~d}_{\mathrm{r}}}-\left(\mathrm{K}_{\mathrm{p}}-1\right) \frac{\sigma_{\mathrm{r}}}{\mathrm{r}}=\frac{\sigma_{\mathrm{c}}}{\mathrm{r}}
$$

Son intégration par la méthode de variation de la constante donne pour $\mathrm{K}_{\mathrm{p}}>1$ :

$$
\sigma_{\mathrm{r}}=-\frac{\sigma_{\mathrm{c}}}{\mathrm{K}_{\mathrm{p}}-1}+\operatorname{ar} \mathrm{K}_{\mathrm{p}}^{-1}
$$

Les relations (3) et (4) permettent de calculer la constante a et d'établir une relation entre $\sigma_{5}$ et $\sigma_{\mathrm{T}}$ :

$$
\begin{gathered}
\sigma_{\mathrm{s}}=-\frac{\sigma_{\mathrm{c}}}{\mathrm{K}_{\mathrm{p}}-1}+\mathrm{a} \mathrm{H}^{K_{\mathrm{p}}^{-1}} \\
\mathrm{~K}_{\mathrm{p}} \sigma_{\mathrm{T}}+\sigma_{\mathrm{c}}=-\frac{\sigma_{\mathrm{c}}}{\mathrm{K}_{\mathrm{p}}-1}+\mathrm{a}\left(\frac{\mathrm{D}}{2}\right)_{\mathrm{p}}^{-1}
\end{gathered}
$$

Cette relation s'écrit :

$$
\mathrm{K}_{\mathrm{p}}\left(\sigma_{\mathrm{T}}+\frac{\sigma_{\mathrm{c}}}{\mathrm{K}_{\mathrm{p}}-1}\right)=\left(\sigma_{\mathrm{s}}+\frac{\sigma_{\mathrm{c}}}{\mathrm{K}_{\mathrm{p}}-1}\right)\left(\frac{\mathrm{D}}{2 \mathrm{H}}\right)_{\mathrm{p}}^{-1}
$$

c'est-à-dire :

$$
\frac{\left(K_{p}-1\right) \frac{\sigma_{s}}{\sigma_{c}}+1}{\left(K_{p}-1\right) \frac{\sigma_{T}}{\sigma_{c}}+1}=K_{p}\left(2 \frac{C}{D}+1\right)^{K_{p}^{-1}} \text { si } K_{p}>1
$$

Le même calcul peut être effectué pour $\mathrm{K}_{\mathrm{p}}=1$ :

$$
\begin{gathered}
\sigma_{\mathrm{s}}=\sigma_{\mathrm{c}} \ln \mathrm{H}+\mathrm{b} \\
\sigma_{\mathrm{T}}+\sigma_{\mathrm{c}}=\sigma_{\mathrm{c}} \ln \frac{\mathrm{D}}{2}+\mathrm{b}
\end{gathered}
$$

d'où :

$$
\frac{\sigma_{s}-\sigma_{T}}{\sigma_{c}}=1+\ln \left(2 \frac{C}{D}+1\right) \quad \text { si } K_{p}=1
$$

$2^{e}$ cas : $\sigma_{1}=\sigma_{\mathrm{r}}$

On a alors :

$$
\begin{aligned}
& \sigma_{\mathrm{T}}=\mathrm{K}_{\mathrm{p}} \sigma_{0}+\sigma_{\mathrm{c}} \\
& \sigma_{\mathrm{t}}=\mathrm{K}_{\mathrm{p}} \sigma_{\theta}+\sigma_{\mathrm{c}}
\end{aligned}
$$

c'est-à-dire :

$$
\sigma_{0}=\frac{\sigma_{T}-\sigma_{c}}{\mathrm{~K}_{\mathrm{p}}} \quad \frac{\mathrm{d} \sigma_{\mathrm{r}}}{\mathrm{d} r}+\frac{\left(\mathrm{K}_{\mathrm{p}}-1\right)}{\mathrm{K}_{\mathrm{p}}} \frac{\sigma_{\mathrm{r}}}{\mathrm{r}}=\frac{-1}{\mathrm{~K}_{\mathrm{p}}} \frac{\sigma_{\mathrm{c}}}{\mathrm{r}}
$$

ce qui donne pour $\mathrm{K}_{\mathrm{p}}>1$ :

$$
\begin{gathered}
\sigma_{\mathrm{s}}=-\frac{\sigma_{\mathrm{c}}}{\mathrm{K}_{\mathrm{p}}-1}+\mathrm{a}^{\prime} \mathrm{H}^{-\frac{\mathrm{K}_{\mathrm{p}}-1}{K_{\mathrm{p}}}} \\
\frac{\sigma_{\mathrm{T}}-\sigma_{\mathrm{c}}}{\mathrm{K}_{\mathrm{p}}}=-\frac{\sigma_{c}}{\mathrm{~K}_{\mathrm{p}}-1}+\mathrm{a}^{\prime}\left(\frac{\mathrm{D}}{2}\right)^{-\frac{\mathrm{K}_{\mathrm{p}}-1}{K_{\mathrm{p}}}}
\end{gathered}
$$

c'est-à-dire :

$\frac{1}{\mathrm{~K}_{\mathrm{p}}}\left(\sigma_{\mathrm{T}}+\frac{\sigma_{\mathrm{c}}}{\mathrm{K}_{\mathrm{p}}-1}\right)=\left(\sigma_{\mathrm{s}}+\frac{\sigma_{\mathrm{c}}}{\mathrm{K}_{\mathrm{p}}-1}\right)\left(2 \frac{\mathrm{C}}{\mathrm{D}}+1\right) \frac{\mathrm{K}_{\mathrm{p}}-1}{\mathrm{~K}_{\mathrm{p}}}$ 
ou encore :

$$
\frac{\left(K_{p}-1\right) \frac{\sigma_{T}}{\sigma_{c}}+1}{\left(K_{p}-1\right) \frac{\sigma_{s}}{\sigma_{c}}+1}=K_{p}\left(2 \frac{C}{D}+1\right)^{\frac{K_{p}-1}{K_{p}}} \text { si } K_{p}>1
$$

Pour $\mathrm{K}_{\mathrm{p}}=1$, on a de même :

$$
\begin{gathered}
\sigma_{s}=-\sigma_{c} \ln \mathrm{H}+\mathrm{b}^{\prime} \\
\sigma_{\mathrm{T}}-\sigma_{\mathrm{c}}=-\sigma_{\mathrm{c}} \ln \frac{\mathrm{D}}{2}+\mathrm{b}^{\prime}
\end{gathered}
$$

c'est-à-dire :

$$
\frac{\sigma_{\mathrm{T}}-\sigma_{\mathrm{s}}}{\sigma_{\mathrm{c}}}=1+\ln \left(2 \frac{\mathrm{C}}{\mathrm{D}}+1\right) \quad \text { si } \mathrm{K}_{\mathrm{p}}=1
$$

\section{ANNEXE IV}

\section{Amélioration de la borne inférieure}

\section{de H.B. MÜLHAUS (cas où $\sigma_{T} \neq 0$ )}

On considère le champ de contrainte de la figure 1 .

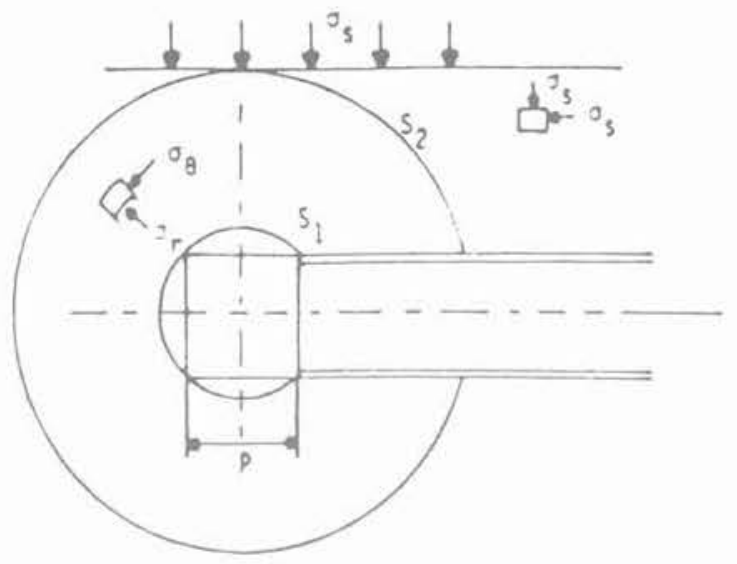

Fig. 1 (annexe IV).

Le champ est uniforme et vaut $\sigma_{\mathrm{s}}$ au-delà de la sphère $\mathrm{S}_{2}$ : il est égal à $\sigma_{\mathrm{T}}$ à l'intérieur de la sphère $\mathrm{S}_{1}$. Dans tout l'espace compris entre les sphères $S_{1}$ et $S_{2}$, le critère est atteint :

$$
\left|\sigma_{1}-\sigma_{3}\right|=2 c^{\prime} \cos \varphi^{\prime}+\left(\sigma_{1}+\sigma_{3}\right) \sin \varphi^{\prime}
$$

c'est-à-dire en exprimant c' en fonction de la résistance en compression simple $\sigma_{c}$ et en faisant l'hypothèse que la contrainte principale majeure est $\sigma_{1}$ :

$$
\sigma_{1}=\mathrm{K}_{\mathrm{p}} \sigma_{3}+\sigma_{\mathrm{c}}
$$

On fait également l'hypothèse que $\sigma$ est à symétrie sphérique entre les deux sphères, ce qui permet de ramener les équations d'équilibre à :

$$
\frac{\mathrm{d} \sigma_{\mathrm{t}}}{\mathrm{dr}}+2 \frac{\sigma_{\mathrm{t}}-\sigma_{0}}{\mathrm{r}}=0
$$

Deux cas peuvent se présenter :

1er cas : $\sigma_{1}=\sigma_{\theta}$

Alors compte tenu de (1), l'équation (2) devient :

$$
\frac{\mathrm{d} \sigma_{\mathrm{r}}}{\mathrm{dr}}+2 \frac{\sigma_{\mathrm{f}}-\left(\mathrm{K}_{\mathrm{p}} \sigma_{\mathrm{f}}+\sigma_{\mathrm{c}}\right)}{\mathrm{r}}=0
$$

soit :

$$
\frac{\mathrm{d} \sigma_{\mathrm{r}}}{\mathrm{dr}}=2\left(\mathrm{~K}_{\mathrm{p}}-1\right) \frac{\sigma_{\mathrm{r}}}{\mathrm{r}}+\frac{2 \sigma_{\mathrm{c}}}{\mathrm{r}}
$$

en intégrant cette équation par la méthode de variation de la constante on obtient pour $\mathrm{K}_{\mathrm{p}}>1$ :

$$
\begin{gathered}
\sigma_{q}=\mathrm{A}(\mathrm{r}) \mathrm{r}^{2}\left(\mathrm{~K}_{\mathrm{p}}-1\right) \\
\mathrm{r}^{2}\left(\mathrm{~K}_{\mathrm{p}}-1\right) \frac{\mathrm{dA}}{\mathrm{dr}}=\frac{2 \sigma_{\mathrm{c}}}{\mathrm{r}} \\
\mathrm{A}(\mathrm{r})=-\frac{\sigma_{\mathrm{c}}}{\mathrm{K}_{\mathrm{p}}-1} \mathrm{r}^{-2\left(\mathrm{~K}_{\mathrm{p}}-1\right)}+\mathrm{a}, \text { soit : } \\
\sigma_{\mathrm{r}}=-\frac{\sigma_{\mathrm{c}}}{\mathrm{K}_{\mathrm{p}}-1}+\mathrm{ar}^{2\left(\mathrm{~K}_{\mathrm{p}}-1\right)}
\end{gathered}
$$

Pour que $g$ soit solution du système d'équations $(2.3 . \mathrm{b}), \quad(2.3 .2 \mathrm{a}), \quad(2.3 .2 \mathrm{~b}), \quad(2.3 .2 \mathrm{c}), \quad(2.3 .2 \mathrm{~d})$, $(2.3 .3 \mathrm{~b})$ de $(\mathrm{Pb} \mathrm{I})$, il reste à vérifier les conditions aux limites :

$$
\begin{aligned}
& \sigma_{s}=-\frac{\sigma_{c}}{K_{p}-1}+\mathrm{aH}^{2\left(K_{p}-1\right)} \\
& \sigma_{T}=\frac{-\sigma_{c}}{K_{D}-1}+a\left(\frac{D^{\prime}}{2}\right)^{2\left(K_{p}-1\right)}
\end{aligned}
$$

D' étant le diamètre de la sphère $S_{1}$ on a : $\mathrm{D}^{\prime 2}=\mathrm{P}^{2}+\mathrm{D}^{2}$

soit :

$$
\frac{P}{D}=\sqrt{\left(\frac{D^{\prime}}{D}\right)^{2}-1}
$$

La relation (2) permet de calculer la constante d'intégration a :

$$
\mathrm{a}=\left(\sigma_{\mathrm{s}}+\frac{\sigma_{\mathrm{c}}}{\mathrm{K}_{\mathrm{p}}-1}\right) \mathrm{H}^{-2\left(\mathrm{~K}_{\mathrm{p}}-1\right)}
$$

La relation (3) donne alors :

$$
D^{\prime 2}=\left[\frac{\sigma_{\mathrm{T}}+\frac{\sigma_{\mathrm{c}}}{\mathrm{K}_{\mathrm{p}}-1}}{\sigma_{\mathrm{s}}+\frac{\sigma_{\mathrm{c}}}{\mathrm{K}_{\mathrm{p}}-1}}\right]^{\frac{1}{\mathrm{~K}_{\mathrm{p}}-1}} \quad(2 \mathrm{C}+\mathrm{D})^{2}
$$

c'est-à-dire en reportant dans (4) :

$\frac{P^{2}}{D^{2}}=\left(2 \frac{C}{D}+1\right)^{2}\left(\frac{\left(K_{p}-1\right) \frac{\sigma_{T}}{\sigma_{c}}+1}{\left(K_{p}-1\right) \frac{\sigma_{s}}{\sigma_{c}}+1}\right) \frac{1}{K_{p}-1}-1$ si $\mathrm{K}_{\mathrm{p}}>1$ 
Le même calcul pour $\mathrm{K}_{\mathrm{p}}=1$, conduit à :

$$
\frac{\mathrm{d} \sigma_{\mathrm{r}}}{\mathrm{d}}=2 \frac{\sigma_{\mathrm{c}}}{\mathrm{r}}
$$

c'est-à-dire :

$$
\sigma_{t}=2 \sigma_{c} \ln r+b
$$

avec :

$$
\begin{aligned}
& \sigma_{s}=2 \sigma_{c} \ln \mathrm{H}+\mathrm{b} \\
& \sigma_{\tau}=2 \sigma_{c} \ln \frac{\mathrm{D}^{\prime}}{2}+\mathrm{b}
\end{aligned}
$$

d'où :

$$
\sigma_{\mathrm{T}}=2 \sigma_{\mathrm{c}} \ln \frac{\mathrm{D}^{\prime}}{2 \mathrm{H}}+\sigma_{\mathrm{s}}
$$

et

$\frac{\mathrm{p}^{2}}{\mathrm{D}^{2}}=\left[\frac{2 \frac{\mathrm{C}}{\mathrm{D}}+1}{\exp \frac{\sigma_{\mathrm{s}}-\sigma_{\mathrm{T}}}{2 \sigma_{\mathrm{c}}}}\right]^{2}-1$

si $K_{p}=1$

$2^{e}$ cas : $\sigma_{1}=\sigma_{r}$

L'équation (2) s'écrit alors :

$$
\left.\frac{\mathrm{d} \sigma_{\mathrm{r}}}{\mathrm{d}_{\mathrm{r}}}+2 \frac{\left(\mathrm{K}_{\mathrm{p}}-1\right)}{\mathrm{K}_{\mathrm{p}}}\right) \frac{\sigma_{\mathrm{r}}}{\mathrm{r}}=-\frac{2 \sigma_{\mathrm{c}}}{\mathrm{K}_{\mathrm{p}} \mathrm{r}}
$$

son intégration pour $\mathrm{K}_{\mathrm{p}}>1$ conduit à :

$$
\sigma_{t}=\frac{\sigma_{c}}{K_{p}-1}+a^{\prime} H^{-2\left(\frac{K_{p}-1}{K_{p}}\right)}
$$

avec :

$$
\begin{aligned}
& \sigma_{s}=\frac{-\sigma_{c}}{K_{p}-1}+a^{\prime} H^{-2} \frac{\left(K_{p}-1\right)}{K_{p}} \\
& \sigma_{T}=\frac{-\sigma_{c}}{K_{p}-1}+a^{\prime}\left(\frac{D^{\prime}}{2}\right)^{-2} \frac{\left(K_{p}-1\right)}{K_{p}}
\end{aligned}
$$

c'est-à-dire :

$$
\frac{\mathrm{p}^{2}}{\mathrm{D}^{2}}=\left(2 \frac{\mathrm{C}}{\mathrm{D}}+1\right)^{2}\left(\frac{\left(\mathrm{K}_{\mathrm{p}}-1\right) \frac{\sigma_{\mathrm{s}}}{\sigma_{\mathrm{c}}}+1}{\left(\mathrm{~K}_{\mathrm{p}}-1\right) \frac{\sigma_{\mathrm{T}}}{\sigma_{\mathrm{c}}}+1}\right)^{\frac{\mathrm{K}_{\mathrm{p}}}{\mathrm{K}_{\mathrm{p}}-1}}-1
$$

si $\mathrm{K}_{\mathrm{p}}>1$
Si $\mathrm{K}_{\mathrm{p}}=1$, on a à résoudre :

$$
\frac{\mathrm{d} \sigma_{\mathrm{r}}}{\mathrm{dr}}=-\frac{2 \sigma_{\mathrm{c}}}{\mathrm{K}_{\mathrm{p}} \mathrm{r}}
$$

On obtient donc le même résultat que dans le cas $\sigma_{1}=\sigma_{\theta}$ en changeant $\sigma_{\mathrm{c}}$ en $-\sigma_{\mathrm{c}}$ :

$$
\frac{\mathrm{P}^{2}}{\mathrm{D}^{2}}=\left[\frac{2 \frac{\mathrm{C}}{\mathrm{D}}+1}{\exp \frac{\sigma_{\mathrm{T}}-\sigma_{\mathrm{s}}}{2 \sigma_{\mathrm{c}}}}\right]^{2}-1 \quad \text { si } \mathrm{K}_{\mathrm{p}}=1
$$

Dans la pratique le $1^{\text {er }}$ cas correspondra à une rupture par effondrement et le $2^{e}$ cas à une rupture par explosion.

Par ailleurs $\mathrm{K}_{\mathrm{p}}=1$ représente le cas d'un sol purement cohérent.

\section{REMERCIEMENTS}

Nous tenons à remercier J. SALENCON pour les conseils qu'il nous a apporté dans la réalisation du travail présenté dans cet article.

\section{BIBLIOGRAPHIE}

ATKINSON J.H., CAIRNCROSS A.M. (1973), Collapse of a shallow tunnel in a MOHR-COULOMB material, Proc. Symp. Role of plasticity in soil mechanics, Cambridge, 13-15 September 1973, pp. 202. 206.

ATKINSON J.H., CAIRNCROSS A.M., JAMES R.G. (1974), Model tests on shallow tunnels in sand and clay, Tunnels and Tunnelling, juillet 1974, pp. 28-32. ATKINSON J.H., BROWN E.T., POTTS M. (1975), Collapse of shallow unlined tunnels in dense sand, Tunnels and Tunnelling, mai 1975.

ATKINSON J.H., POTTS D.M.(1977), Stability of a shallow circular tunnel in cohesionless soil, Géotechnique $27, n^{\circ} 2$, pp. 203-215.

ATTEWELL P.B., BODEN J.B.(1971), Development of stability ratios for tunnels driven in clay, Tunnels and Tunnelling, mai 1971, pp. 195-198.

BROMS B.B., BENNERMARK H. (1967), Stability of clay at vertical openings, Journal of the Soil Mechanics and Foundations Division, A.S.C.E., S.M.L, janvier 1967, pp. 71-94.

COUSSY O., SALENÇON J. (1979), Analyse de la stabilité des ouvrages en terre par le calcul à la rupture, Annales des Ponts et Chaussées, 4e trimestre 1979, $35 \mathrm{p}$. 
CUNHA A.P. (1983), Analysis of advancing tunnels in rock, International Congress on rock mechanics, Melbourne, mai 1983, Balkema vol. 2, pp. 153-157.

DAVIS E.P., GUNN M.J., MAIR R.J., SENEVIRATNE H.N. (1980), The stability of shallow tunnels and underground openings in cohesive material, Géotechnique $30, n^{\circ} 4$, pp. 397-416.

MANDEL J., HABIB P., D'ESCATHA Y., HALPHEN B., LUONG M.P., ZARKA J. (1974), Étude théorique et expérimentale de la stabilité des cavités souterraines, Symposium franco-polonais, Problèmes de Rhéologie et de Mécanique des Sols, Nice.

MULHAUS H.B. (1985), Lower bound solutions for circular tunnels in two and three dimensions, Rock Mechanics and Rock Engineering 18, pp. 37-52.

PECK R.B. (1969), Deep excavations and tunnelling in soft ground, Proceedings of the VIlth International Conference on Soil Mechanics and Foundation Engineering, Mexico 1969, Balkema vol. 3, pp. 225-290. PERA J. (1984), Présentation générale des boucliers à pression de boue, Comptes-rendus des Journées d'Études Internationales, A.F.T.E.S., Lyon, novembre 1984, pp. 3-7.

PROCTOR R.V., WHITE T.L. (1977), Earth tunnelling with steel supports, ch. 4 : Mechanics of squeezing and swelling.

ROMO M.P., DIAZ C.M. (1981), Face stability and ground settlement in shield tunneling. Proc. Xth Int. Conf. Soil Mech. Found. Engng., Stockholm, juin 1981, Balkema vol. 1, pp. 359-360.

SALENÇON J. (1983), Calcul à la rupture et analyse limite, Presses de l'École Nationale des Ponts et Chaussées, Paris 1983 , p. 366.

SCHOFIELD A.N. (1980), Cambridge geotechnical centrifuge operations, Geotechnique $30, \mathrm{n}^{\circ} 3$, pp. 227-268.

SMOLTCZYK V., HOLZMANN P. (1969), Earth pressure reduction in front of a tunnel shield, Proc. VIIth Int. Conf. Soil Mech. Found. Engng. Mexico 1969, Balkema vol. 2, pp. 478-481.

\section{ÉCOLE NATIONALE DES PONTS ET CHAUSSÉES FORMATION CONTINUE Sessions de formation Géotechnique, matériaux structures Routes, ouvrages d'art}

Les matériaux dans les pays en développement Logiciels pour les calculs BAEL et BPEL Les enrobés à chaud Les eurocodes et la normalisation européenne Construire en terre : l'avenir du passé

$\begin{array}{ll}7 \text { au } 10 \text { juin } & \text { Paris } \\ 8 \text { et } 9 \text { juin } & \text { Paris } \\ 14 \text { au } 17 \text { juin } & \text { Angers } \\ 21 \text { au } 23 \text { juin } & \text { Paris } \\ 27 \text { au } 29 \text { septembre } & \text { Paris }\end{array}$

Pour toute information, s'adresser à l'E.N.P.C./D.F.C.A.I., 28, rue des Saints-Pères, 75007 PARIS. Tél. 16 (1) 42.60.34.13 (Christine ROSE). 